\title{
Diversité culturelle et diversité biologique : une approche critique fondée sur l'exemple brésilien
}

\author{
Florent Kohler \\ Anthropologue, CREDA, UMR 7227, CNRS/Université Paris 3, Paris, France
}

\begin{abstract}
La Convention sur la diversité biologique (CDB), en liant la diversité culturelle à la diversité biologique, a permis aux «populations autochtones et locales » de faire entendre leur voix et d'accéder à une certaine reconnaissance sur la scène internationale. Les savoirs locaux sont alors devenus l'objet d'enjeux politiques ouvrant sur la revendication de droits fonciers et citoyens. À partir de situations observées en Amazonie, l'auteur propose une lecture distinguant ce qui relève de la rhétorique - le discours étant le matériau privilégié par les anthropologues - et la réalité des pratiques, pour se demander si les modes de gestion traditionnels de la nature sont forcément générateurs de biodiversité et peuvent servir d'outils pour les politiques contemporaines de conservation. Il interroge en cela le rôle des cosmologies natives dans la survie des écosystèmes et les postures théoriques de l'écologie culturelle et des ethnosciences. Florent Kohler ouvre ainsi un débat passionné sur la vision culturalisée de la nature, dont la revue se fait l'écho en publiant deux commentaires à la suite de son texte et qu'elle prolongera dans le numéro suivant en lui donnant un droit de réponse.
\end{abstract}

\section{Mots-clés :}

diversité culturelle ; biodiversité ; peuples indigènes ; populations traditionnelles ; Amazonie brésilienne

\section{Keywords:}

biocultural diversity;

Native people;

traditional

populations;

Brazilian Amazon

\begin{abstract}
Résumé - Les documents préparatoires au sommet de Johannesburg de 2002 portaient l'accent sur les corrélations entre diversités culturelle et biologique. Une étude critique de ces corrélations montre que l'argument se fonde sur des analogies et ne peut dès lors prétendre au rang de concept opératoire. Afin d'appréhender plus finement ces relations, nous proposons quelques éléments pour une heuristique des représentations des populations locales concernant leur environnement, en distinguant les discours contextuels et cosmologiques, et en prônant une meilleure intégration de l'anthropologie dans les études pluridisciplinaires.
\end{abstract}

\begin{abstract}
Biocultural diversity: a critical approach based on the Brazilian model. The preparatory documents for the 2002 Johannesburg Summit focused on correlations between cultural and biological diversity. A critical study of these correlations shows that the argument is based on analogies and cannot consequently claim to a valid operative concept. In order to better understand these relationships, we suggest a heuristic representation of local populations which would include their environment while distinguishing contextual and cosmological views and advocate for a better integration of anthropology in multi-disciplinary studies. Our point is that public policies aiming at protecting biodiversity should be maintained as a distinct objective, and that it should not be assumed that traditional societies are the ideal keepers of ecosystems or endangered species.
\end{abstract}

\begin{abstract}
«Si gênant que ce soit de l'admettre, la nature, avant qu'on songe à la protéger pour l'homme, doit être protégée contre lui. [... ] Le droit de l'environnement, dont on parle tant, est un droit de l'environnement sur l'homme et non un droit de l'homme sur l'environnement. »
\end{abstract}

Lévi-Strauss (1983, p. 375).

On peut s'étonner que les positions de plus en plus tranchées de Lévi-Strauss en faveur d'un «humanisme bien conçu » (Lévi-Strauss, 1971), sa vision toujours plus pessimiste d'une humanité échappant à tout contrôle et

Auteur correspondant : florent.kohler@gmail.com «saccageant» (ibidem) la planète qui lui a donné vie, n'aient pas fait l'objet de l'attention que lui vaut le reste de son œuvre. Tout se passe comme si l'anthropologue, le philosophe et l'esthète méritaient une considération, tandis que ses préoccupations écologiques, plus affirmées à la fin de son parcours, relevaient d'un autre domaine de sa pensée, éloignée des humanités, en tant qu'ensemble de sciences morales. Quelle est la position des anthropologues à ce sujet? Tant Descola (2005) que Latour (1999) ou Ingold (1994) et Viveiros de Castro (2008) - ce dernier, par ses positions publiques en faveur de la protection de 
l'Amazonie en l'état - se sont souciés d'inclure les nonhumains dans des réseaux de relation. Mais, lorsqu'il s'agit d'aller au bout de cette logique, les obstacles méthodologiques et déontologiques sont tels qu'il semblerait préférable de se limiter à une proposition théorique. Il convient de s'interroger sur le malaise que pourrait susciter une telle proposition : longtemps subordonnée à l'expansion coloniale, l'ethnologie a joué, à partir des années cinquante, un rôle-clé dans la prise de conscience d'une unicité humaine et dans le rejet des modèles de cultures hiérarchisées. Ce faisant, les mêmes ethnologues ont contribué à accentuer le fossé creusé par le "grand partage » (Latour, 1991 ; Schaeffer, 2007) entre l'humanité et le reste du vivant, posant l'homme comme produit de sa ou ses cultures, hors de tout processus darwinien (Stoczkowski, 1994), certains allant jusqu'à adopter un anti-évolutionnisme de principe (Gras, 2004).

Si la corporation défend unanimement l'idée de territoires alloués spécialement à des populations autochtones ou traditionnelles afin qu'elles y perpétuent leur mode de vie et leurs traditions - invoquant leurs pratiques ancestrales et leur rôle de gardiens des équilibres écologiques (Posey, 1985 ; Balée, 1994 ; Diegues, 1996) -, elle s'élève presque aussi unanimement (si l'on excepte Cunha et Almeida, 2000, ou Posey et Dutfield, 1996) contre toute tentative de contractualiser ces populations au motif qu'elles peuvent, étant chez elles, agir comme bon leur semble. L'argument écologique mis en avant en début de processus est ultérieurement battu en brèche par la dénonciation d'un "gouvernement de la nature » qui voudrait pérenniser la protection des milieux au nom d'une vision occidentale (Diegues, 1996 ; Nadasdy, 2005) de la préservation.

$\mathrm{Au}$ sein de la corporation, le discours dominant semble désormais enraciné, qui pose comme allant de soi que les cosmologies "natives " sont respectueuses de l'environnement et génératrices de biodiversité. Ce discours, nous souhaitons l'aborder et en proposer une critique fondée sur la littérature existante et sur nos différents terrains brésiliens ${ }^{1}$, en contexte indigène ou traditionnel, selon une problématique de "durabilité ». Pour l'heure, et selon cette idée que, parmi les solutions proposées, certaines s'avéreront non viables après que des dégâts irréversibles auront été causés - comme ce fut le cas dans le Parc national du mont Pascal au Brésil (Kohler, 2008) ou dans celui des Galápagos

\footnotetext{
1 Entre 2001 et 2009, une vingtaine de terrains d'une durée de trois semaines à trois mois : terres indigènes Pataxó (Bahia), terres indigènes de l'Uaçá (Amapá), communautés traditionnelles du Parc national Cabo Orange, Réserves de développement durable d'Iratapuru (Amapá) et Mamirauá (Amazonas) et coopératives d'Ouro Preto d'Oeste (Rondônia), ces trois derniers terrains ayant été effectués dans le cadre du programme DURAMAZ, coordonné par le CREDA (CNRSUniversité Paris 3) et financé par l'ANR.
}

(Grenier, 2008) -, l'approche anthropologique doit s'imposer, parmi d'autres approches, comme celle qui aborde frontalement la question des motivations et représentations individuelles. Nous abordons cette question sous deux angles : le premier porte sur la nécessité d'une heuristique des représentations faisant la part de ce qui relève de la cosmologie et de ce qui renvoie à une rhétorique de mobilisation ; l'autre porte sur l'insertion des anthropologues au sein d'équipes de chercheurs d'autres disciplines ${ }^{2}$.

\section{Nouveau paradigme ou pétition de principe?}

L'objet de cette première partie est de pointer le danger qui consiste à subordonner l'écologique au culturel, après avoir longtemps sacrifié l'un et l'autre au pragmatisme économique. Au cours des années 1990 s'est opérée une alliance objective entre environnementalistes et indigénistes (Morin, 1992 ; Conklin et Graham, 1995; Ferreira, 1999; Chapin, 2004; Dove, 2006; DumoulinKervran, 2006; Barretto Filho, 2006) grâce à laquelle s'est popularisée l'idée selon laquelle la diversité culturelle, exprimée par une diversité d'usages des ressources naturelles, jouait un rôle majeur dans le respect des équilibres écologiques. La contribution des ethnologues (Rappaport, 1968) fut essentielle à l'élaboration de cette ligne directrice de la pensée de l'Unesco telle qu'elle s'est exprimée au sommet de Johannesburg en 2002, selon deux axes majeurs : "promouvoir la pluralité des cultures, dans toutes leurs expressions, et lutter contre la perte de la diversité biologique»(Unesco, 2003, p. 10). Ce sommet ayant fait suite à la conférence de Rio en 1992, on constate aisément que les urgences écologiques ont cédé la place, en dix ans à peine, à des considérations plus nuancées, plus anthropocentrées, par un phénomène progressif de «culturalisation » de l'environnement : "En mettant l'accent sur la "diversité durable", nous reconnaissons que les être humains appartiennent à l'univers biologique, tout en étant la seule espèce sur terre qui a le privilège d'intervenir sur lui par la création de diverses formes de culture dans le temps et dans l'espace. [... ]. Ainsi, la diversité culturelle doit être vue comme un gage de la biodiversité. » (Ibid., p. 8.)

Parce que ces deux notions sont étroitement mêlées, elles sont conceptualisées de la même manière : 1'unicité dans la multiplicité, un ensemble formé par de multiples

\footnotetext{
${ }^{2}$ Une première version de cet article formait un chapitre de mon habilitation à diriger des recherches, intitulée «L'animal qui n'en était pas un ». Il résulte du constat suivant : bien souvent, les anthropologues ne maîtrisent pas les connaissances biologiques, zoologiques ou botaniques, non plus que les processus biocénotiques, qui leur permettraient d'établir en toute certitude que les cosmologies qu'ils décrivent correspondent à une réalité sur les terrains qu'ils étudient.
} 
parties (Harmon, 1996). L'environnement est à la fois «culturel et biologique », et la biodiversité intègre toutes les variations génétiques ou phylogénétiques produites par l'élevage et l'agriculture, les multiples races de ruminants, de volailles ou de variétés horticoles, manifestations de la «force créatrice » de l'humanité (Unesco, 2003, p. 9). En prenant en compte la multiplicité des variétés domestiques de plantes et d'animaux, l'Unesco renforce le lien entre diversité culturelle et diversité biologique, puisque ces variétés d'élevages se situent à l'interface de la culture et du monde naturel : produites par une culture donnée, intégrant toutefois le cycle naturel de diversification des espèces. La relation s'exprime donc par des usages, des pratiques, voire des paysages :

«Les populations ont également créé et continuent à entretenir des paysages culturels afin de maintenir des valeurs écologiques et culturelles spécifiques. Les paysages naturels variés, créés et entretenus par les aborigènes australiens, à travers leur utilisation ingénieuse $\mathrm{du}$ feu, en sont un exemple parmi les plus connus. Il n'est jusqu'à la forêt tropicale amazonienne, considérée par nombre de personnes comme l'ultime expression de l'espace primitif, qui n'ait été façonnée au cours des millénaires par les interventions délibérées des peuples autochtones. » (Ibid., p. 9.)

On constate un vaste effort conceptuel permettant d'associer les deux faces d'une même médaille, selon le discours officiel : la diversité des cultures est garante, mais aussi productrice, de diversité biologique. On ne saurait donc, selon de telles prémisses, dissocier la question de la biodiversité de celle de la pauvreté, et c'est en luttant contre celle-ci qu'on préservera celle-là (Unesco, 2003). En désorganisant les systèmes traditionnels de production, en provoquant une prédation accélérée ou en favorisant l'exode rural, la pauvreté serait ainsi l'un des multiples corollaires de la crise environnementale. Ces propositions ambitieuses et généreuses comportent cependant deux dangers, qui sont aussi des glissements idéologiques.

Le premier glissement est que la biodiversité ne peut être considérée et conceptualisée selon le même modèle que les cultures humaines. Bien entendu, on peut envisager la biodiversité comme un ensemble diversifié d'espèces et de biotopes, fonctionnant sur le modèle du « tous égaux en principe». Mais la biodiversité n'existe pas pour la contemplation de l'homme et sa satisfaction intellectuelle : les espèces interagissent entre elles, interagissent avec les milieux, répondent à des changements, et les écosystèmes valent non seulement par leur diversité, mais aussi par leur capacité à agir comme des organismes complexes. Car la disparition d'un élément appartenant à un ensemble symbiotique ne peut être conceptualisée de la même manière que la disparition d'une langue humaine (Harmon, 1996 ; Harmon et Maffi, 2002) ; elles ne relèvent pas du même modèle et il est vain de les comparer, même si la métaphore est séduisante; la diversité biologique, selon cette métaphore, est perçue comme la somme de ses parties (à l'instar de la diversité culturelle). Or, la disparition d'un élément de la biodiversité peut entrâ̂ner, à plus ou moins brève échéance, le déséquilibre du système - il en va ainsi des systèmes symbiotiques de pollinisation et de dispersion des semences par des vertébrés ou des invertébrés -, cependant que la disparition d'un élément de la diversité culturelle, pour regrettable qu'elle soit, ne menace pas la survie de l'humanité en tant qu'espèce.

Ma première remarque est que les discours assimilant les diversités culturelle et biologique relèvent davantage de pétitions de principe (mode par lequel un sommet international est susceptible de cadrer les débats), et suggèrent en réalité des rapports de simple analogie : nous nous trouvons dans le cas de figure décrit par Descola (2005) selon lequel le monde qui nous entoure, et tout ce qu'il contient, constitue la métaphore de la diversité humaine ; il s'agit d'une catégorisation et non d'un fait, et l'humanité demeure la mesure de toute chose.

La répétition incessante des mêmes propositions (par exemple Unesco, 2003) illustre leur faible productivité. Le flottement conceptuel se retrouve dans l'intervention d'Appadurai (2003) au même Sommet, qui hésite entre une relation « $d$ 'homologie » et l'affirmation répétée que la diversité humaine est une «garantie puissante » de la biodiversité, au nom d'une "vision plus large du développement durable» (ibid., p. 16) : «Les êtres humains sont les acteurs principaux de ces équilibres, et si leur diversité s'appauvrit, il en va de même du trésor de conceptions morales reliant le bien-être moral et le bienêtre matériel. Ainsi, la diversité culturelle est une garantie puissante de la biodiversité. »

Le deuxième glissement idéologique repose sur cette idée que la diversité culturelle est un gage du maintien de la diversité biologique; le pas trop vite franchi consiste à affirmer que la première est la condition de la deuxième, voire qu'elle la génère. Il s'agit là d'un point polémique dans la mesure où cette idée a surgi dans l'œuvre d'ethnologues appartenant à l'école dite d'écologie culturelle née aux États-Unis. L'ouvrage de Balée (1994), devenu une référence, est exemplaire à cet égard, puisqu'à partir d'un exemple localisé, il fournit les bases conceptuelles qui permettent d'élargir sa proposition à l'ensemble amazonien : l'Amazonie serait le résultat du travail de l'homme, de " générations d'horticulteurs », et devrait donc être considérée comme un paysage. Les implications sont multiples : dans la lutte commune, mais délicate, que mènent groupes de pression environnementaux et groupes autochtones et leurs alliés, cette thèse place la balle dans le camp des autochtones, en termes de priorité et d'argumentation. C'est le sens de la question posée par Appadurai (2003, p. 18) : «Les populations autochtones peuvent-elles être encouragées à se faire une 
place sur le marché mondial sans pour autant sacrifier leurs cosmologies qui sont, fondamentalement, respectueuses [inherently ecofriendly] de l'écosystème? »

Pourquoi est-il préférable de parler de corrélation plutôt que d'interdépendance? Parce que, contrairement à ce qu'affirme Appadurai, les cosmologies ne sont pas toutes, "fondamentalement », respectueuses des écosystèmes (Pinton et Grenand, 2007). Quand elles le sont, c'est souvent après avoir commis un certain nombre de «boulettes ", comme par exemple d'exterminer les mégafaunes d'Europe, d'Amérique, d'Océanie, de Madagascar, de transformer le milieu méditerranéen par la conversion de forêts tempérées en garrigues (surexploitation - en particulier par l'industrie navale antique - et surpâturage) ou de supprimer, de-ci de-là, tout couvert végétal (Tudge, 1996 ; Broswimmer, 2003; Hayashida, 2005; Diamond, 2006 ; Kelly et Prasciunas, 2007). Le cas de l'Australie est fort bien documenté (Gifford et al., 2005) ; ailleurs, si la question de la responsabilité humaine n'est pas tranchée, l'indice de probabilité est fort élevé (Krech, 1999; True et al., 2005 ; Steadman et al., 2005; Surovell et al., 2005). Quand ce n'est pas l'homme lui-même qui est en cause, ce sont ses animaux domestiques ou familiers : chien, chat, porc, mouton, rat (Hunt et Lipo, 2010), mangouste, comme à Hawaï, où $70 \%$ de la faune aviaire a disparu en deux temps (Chatterjee, 1997). On estime ainsi à $25 \%$ du total de l'avifaune mondiale le taux d'espèces disparues $\mathrm{du}$ fait des premières migrations humaines dans les îles du Pacifique, il y a 1000 à 2000 ans (Barbault, 2005).

La survie des écosystèmes n'est donc pas uniquement affaire de cosmologie, sauf à considérer, ce qui est raisonnable, que leur destruction l'est aussi. La capacité des faunes et flores à résister aux premiers chocs, puis aux espèces invasives, est aussi un facteur-clé : c'est la capacité de l'environnement à s'adapter à l'homme, et non l'inverse, qui devrait retenir notre attention. Cette capacité va au-delà de la résilience pour embrasser des phénomènes tels que les changements comportementaux, la reconversion alimentaire ou l'adaptation au milieu urbain. Quant au potentiel destructeur que recèle toute cosmologie humaine, il est totalement passé sous silence (ou attribué au seul expansionnisme de la civilisation occidentale) par l'écologie culturelle américaine, dont le flambeau fut repris par les constructionnistes brésiliens, notamment Diegues (1996), leur chef de file. Cette vision culturalisée de la nature comporte deux aspects : le premier dérive de l'inclusion d'espèces animales et végétales domestiquées dans la biodiversité ; la confusion est volontairement maintenue entre approche quantitative (par exemple, le nombre de races de vaches présentes en France) et mode de fonctionnement des écosystèmes. Le mouton n'intègre pas, dans la réalité, la chaîne alimentaire du loup, ni le bœuf celle du jaguar, pour la bonne raison qu'on commence tout élevage par l'extermination des prédateurs susceptibles de l'affecter - le cas de l'ours pyrénéen constituant un cas d'école (Mermet et Benhammou, 2005). Les multiples variétés de manioc, d'igname ou de taro (Caillon et Degeorges, 2005) ne portent pas avec elles la promesse de systèmes symbiotiques où interviendraient arthropodes et champignons, puisque la lutte biologique est la condition sine qua non de toute exploitation. Sans parler de possibles dérives visant à inclure les organismes génétiquement modifiés dans la biodiversité culturelle, puisqu'ils sont le fruit d'une tradition scientifique solidement attestée dans la culture occidentale.

Le deuxième aspect mis en avant par les approches ethnoécologiques est que le principe de la culture sur brûlis amazonienne, ou la chasse par brûlis des Aborigènes (pour reprendre les exemples cités dans les travaux préparatoires au sommet de Johannesburg), s'il défait un écosystème original, permet à de nouvelles dynamiques de se mettre en place : plantation d'arbres fruitiers favorisant la concentration de singes et autres espèces opportunistes, ouverture de clairières permettant l'apparition d'espèces non arbustives et, dans le cas australien, l'accélération d'un processus de germination qui, à l'état naturel, a besoin du passage d'un incendie pour se développer. Cette vision n'est pas fausse en soi, et s'avère probablement vraie en de multiples endroits, comme le montrent nombre de travaux (en particulier Balée, 1994) avec pour réserves que ces travaux se concentrent sur l'existant et ne prennent donc pas en considération un état antérieur qui n'est que supposé.

Mais cette vision devient fausse à partir du moment où elle est tenue pour règle générale et où elle confond deux ordres de biodiversité : la biodiversité relative et la biodiversité absolue. Un bon exemple nous a été donné par l'étude paléozoologique de Vigne (1999) menée en Corse. La Corse possédait, avant l'arrivée de l'homme, une faune endémique de mammifères, reptiles, amphibiens et oiseaux. Les premières vagues de populations humaines se sont produites au Néolithique, avec le cortège habituel d'espèces domestiques et familières : chien, mouton, porc, vache, puis chat, cheval, mais aussi souris, belette, loir, hérisson (d'introduction récente), ours (idem), volaille, pie, étourneau, moineau, etc. La totalité des espèces endémiques de mammifères a disparu à la suite de ces invasions. En termes relatifs, la biodiversité en Corse est supérieure aujourd'hui (avec 26 espèces de mammifères contre 17 auparavant). Mais cette biodiversité n'est pas propre à la Corse : elle est continentale. La faune insulaire, qui ajoutait à la biodiversité totale existant sur Terre, a diminué : en termes absolus, donc, le solde est négatif. Toute affirmation selon laquelle « la biodiversité augmente à l'arrivée de l'homme » doit donc être soigneusement pesée (Hames, 2007, p. 55), sauf à dire que la construction d'une ville en plein désert, avec ses chiens, chats, rats, pigeons et poissons rouges, est une contribution humaine à la création et au maintien 
d'écosystèmes, ou à prétendre que l'extinction des mégafaunes américaines n'est qu'un détail au regard de la biodiversité entretenue par les systèmes agroforestiers mis en place par les populations précolombiennes.

\section{Sur la méthode anthropologique}

Comme l'observe Ferreira (2004), les mouvements socio-environnementalistes brésiliens, en prenant position pour l'arrêt des politiques d'exclusion humaine dans les stratégies de conservation, ont «abouti à idéologiser le débat académique, en diminuant l'impact scientifique des recherches visant à étudier effectivement les caractéristiques de l'usage des ressources naturelles par les populations résidant dans des aires biogéographiques caractérisées par une haute biodiversité ». Or, c'est précisément le rôle joué par les anthropologues dans l'empowerment des populations rurales néotropicales qui rend leur position délicate à l'heure de prendre leur part dans ce débat, et cela, pour deux raisons : l'une concerne la praxis scientifique; l'autre, la déontologie. Il convient donc de soulever la question de l'avenir des aires protégées, habitées ou non - et nous entendons ici «aires protégées » au sens générique, incluant, par exemple, les terres indigènes dès lors qu'elles intègrent, au Brésil, le système dit de «mosaïque de zones de préservation »face à une croissance humaine qui sera toujours, en fin de compte, tenue pour prioritaire (Ehrlich et Pringle, 2008).

\section{Heuristique des représentations}

D'abord, de quels outils disposons-nous pour analyser les discours locaux de manière critique, dans un contexte de mobilisation politique - avec ce que cela implique de propos convenus et stéréotypés? Et, dès lors que l'enquête anthropologique repose essentiellement sur un ensemble de représentations qui nous sont livrées par la parole de nos informateurs, comment déployer une heuristique du discours qui permette d'appréhender les différentes strates de ces représentations, y compris celles qui demeurent informulées? Nous répondrons à cela par quelques éléments d'une théorie des stéréotypes conçue précisément pour appréhender des représentations complexes (Kohler, 2007).

Proposons comme angle d'attaque la représentation cartographique, qui constitue un bon indice de la manière dont l'espace est appréhendé. Sur la carte dessinée par un Indien Galibi-Marworno de l'Uaçá (Nord de l'Amapá) [Fig.], les écosystèmes de la réserve sont minutieusement figurés : savanes inondables, savanes sèches, forêts inondables, hautes futaies. . Une cartographie écosystémique paraît congruente avec un ensemble de pratiques suggérant un usage judicieux des ressources à l'époque où elles se présentent : poisson en saison sèche, lorsque le lit des fleuves se resserre et que les marais disparaissent; gibier en saison humide, lorsque les animaux se concentrent sur des îlots ou dans les hautes futaies. La territorialité (et le discours mythique qui l'accompagne) présente manifestement, dans l'Uaçá, une configuration qui expliquerait la bonne conservation des ressources et des systèmes naturels. Peut-on dès lors considérer qu'il existerait, ici, une «inclusion sociale » des principaux êtres vivants (Viveiros, 2002), interdisant les prélèvements excessifs, une telle inclusion caractérisant la pensée animiste?

Dans 1’Uaçá, seuls les pajés (nom local attribué aux chamans) ont accès aux lacs enchantés, eux seuls exercent le pouvoir de médiation entre les hommes et les espritsmaîtres, dits karuãna ; ils se posent donc, apparemment, en garants de l'équilibre entre peuplement humain et sauvegarde des ressources, dont ils pourraient, selon leurs capacités, libérer ou restreindre l'accès. Mais l'idée que la forêt ou certaines espèces animales ou végétales pourraient s'éteindre est contredite par la foi dans le pouvoir des Mères de repeupler les lieux dévastés. Pour expliquer la disparition puis la réapparition du Caïman noir (Melanosuchus niger), un pajé évoque l'enfermement de leur souche par la Mère des caïmans dans une prison dont les barreaux et le cadenas commencent à s'éroder. Cette optique, relevait Pierre Grenand (in Scheps, 1993), n'implique pas que des mesures de restrictions s'imposent à la collectivité. La régulation des flux végétaux et animaux par l'intervention des esprits-maîtres s'oppose ainsi frontalement à l'opinion et à la politique même des organes de protection de l'environnement, qui placent la responsabilité humaine au premier plan.

De telles données ne nous permettent donc pas d'appréhender la totalité du système de relations entre humains et monde naturel, d'une part parce qu'il se focalise sur la personne du chaman, fonction spécialisée s'il en est, d'autre part parce qu'il se cantonne à un registre explicatif où les humains ne sont que secondairement agents. Or, l'observation de la réalité quotidienne dévoile des manifestations d'hostilité générale à l'égard de la faune non comestible : serpents (évidemment), mais aussi oiseaux, petits mammifères, batraciens, insectes sont tués, ou à tout le moins houspillés. Cette attitude n'est évidemment pas l'apanage des Galibi-Marworno, elle est universellement répandue, mais il nous intéresse de comprendre l'enracinement local de ces pratiques. La description que nous a faite un rezador (guérisseur par la prière dite pota) de ses fonctions nous permet de mieux comprendre l'enjeu du rapport homme-animal :

«Quand un enfant prend peur d'un animal [... ], il faut bénir au nom de l'animal. [...] Si on ne prie pas pour cet enfant, l'animal peut le tuer, le tuer vraiment, car ces animaux de la forêt ne plaisantent pas, quand il prend un enfant il ne le lâche plus, il le prend carrément jusqu'à créer une faiblesse et l'enfant meurt. Les bêtes sont méchantes [Os bichos 

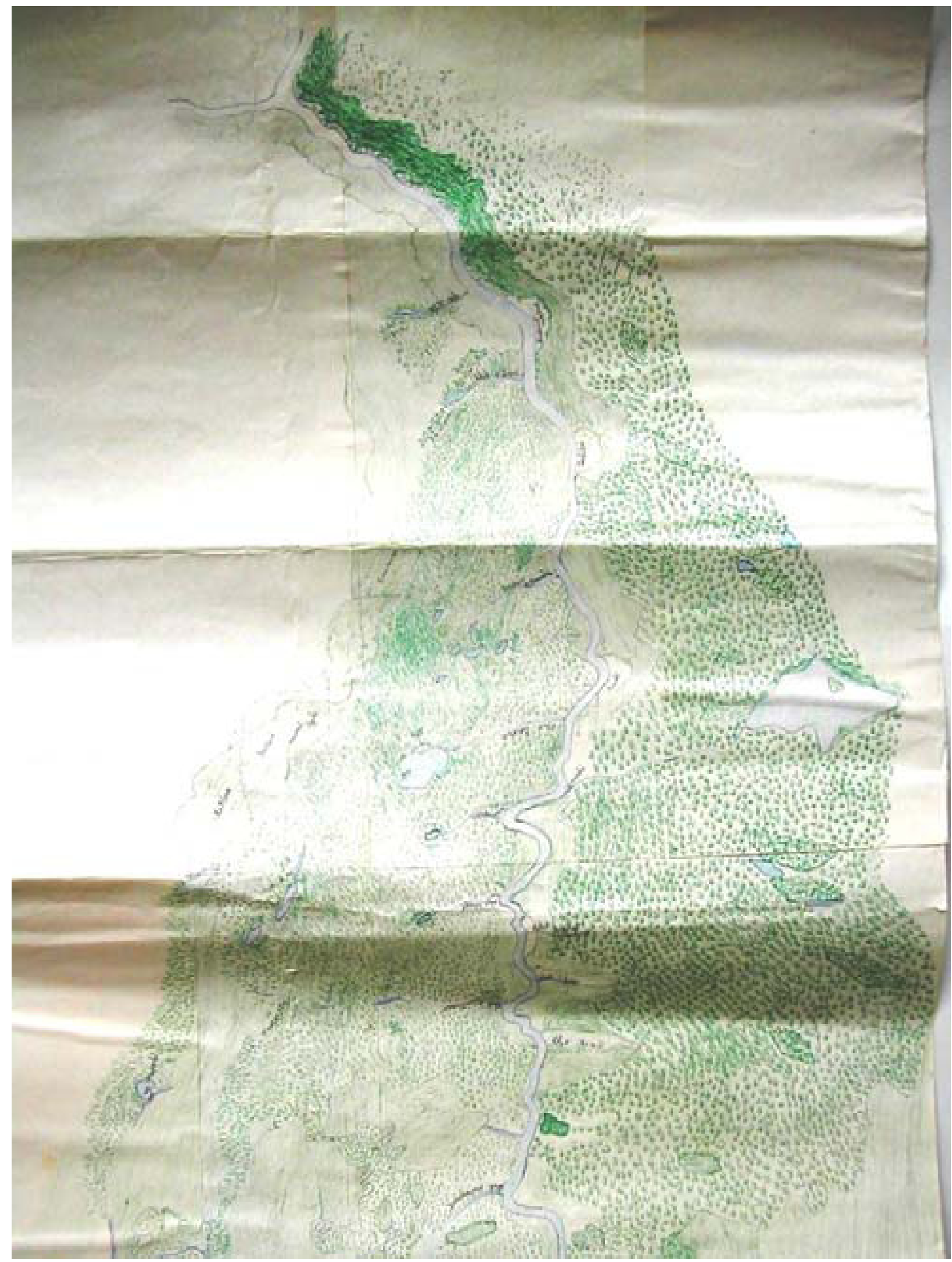

Fig. . Carte dessinée par un Indien Galibi-Marworno (terres indigènes Uaçá) à la demande de l'anthropologue Lux Vidal. 
são malignos] [... ]. Moi je n'ai jamais été épouvanté par une bête, mais un de mes enfants en est mort. »

«Les animaux sont méchants » : nous ne déchiffrerons pas ici les attendus cosmologiques d'une telle assertion, bien qu'elle suggère que les enfants, qui sont les premières victimes, seraient des êtres entre-deux-mondes, des créatures intermédiaires susceptibles de basculer dans l'animalité faute de socialisation adéquate. Le résultat concret en est l'affirmation nette d'une hostilité intrinsèque de la nature à l'égard des hommes, représentation qui s'exprime, ailleurs, par les rites de neutralisation du sang (Clastres, 1975 ; Álvares, 1992).

Il convient donc d'attirer l'attention sur ces représentations qui relèvent de l'ordre cosmologique, et sont évidemment incompatibles avec les discours convenus sur "l'Indien en harmonie avec la nature ", toujours " respectueux des êtres vivants ». Or, ces deux ordres de discours coexistent pour la raison simple qu'ils ne se déploient pas dans le même registre et n'appartiennent pas à la même sphère de représentations. Pour illustrer notre propos, prenons l'exemple d'une phrase récurrente chez les populations, indigènes ou traditionnelles, dès lors qu'elles évoquent leurs pratiques cynégétiques : «je ne chasse que pour manger » ou «j'étais allé chasser pour nourrir ma famille », et observons les conditions d'exercice d'une telle chasse « alimentaire».

Le verbe "chasser», qui revêt un tel poids idéologique lorsqu'il s'agit de territoire amérindien ou «traditionnel », est systématiquement euphémisé (dans l’Uaçá comme ailleurs) lorsqu'un Blanc se trouve présent, sous la forme déclinable à l'infini du «juste pour manger». Or, cette affirmation est contredite par la structure commerciale qui existe dans chaque village, où le prix à la pièce ou au kilo de chaque type de gibier, dans l'Uaçá, est affiché et validé par l'Assemblée des peuples indigènes de l'Oyapock (mais le même principe est appliqué tant chez les Pataxó de Bahia que chez les ribeirinhos ${ }^{3} \mathrm{~d}^{\prime}$ Iratapuru). Le commerce de viande de brousse est donc une activité économique non négligeable, et il est courant que les chasseurs partent en expédition avec une liste de commandes. La chasse - ou la pêche - ne repose pas sur le principe de la mesure dans les prélèvements, sauf coercition ; elle obéit à des facteurs tels que le temps et l'équipement. Ce dont dépend en ultime instance le nombre de bêtes abattues, c'est du nombre de cartouches que l'on emporte avec soi ; encore faut-il vouloir le constater.

Que penser, dès lors, de cette affirmation répétée : «je ne chasse que pour manger »? Elle relève d'une phraséologie imposée, le discours de la durabilité, qui est le produit des médiations multiples qui s'opèrent dans

${ }^{3}$ Les ribeirinhos (" riverains") ou "populations ribeirinhas » désignent les habitants des bords des fleuves et rivières d'Amazonie, vivant d'horticulture, de pêche et d'extraction de produits végétaux et animaux. Les ribeirinhos sont inclus dans la catégorie plus vaste des "populations traditionnelles ». le champ de la négociation (West et al., 2006). Cette négociation peut être perçue comme « interethnique», mais elle est évidemment, et avant tout, politique. Les discours qui s'y réfèrent se déploient donc dans un registre idéologique, activé par la mobilisation contextuelle.

Il ne s'agit pas ici d'opposer une idéologie environnementaliste à une autre, indigéniste, autochtone ou « ethnophile » (Belleau, 2007), mais de reconsidérer ce que sont les éléments pertinents d'une enquête. Si l'on se consacre au chamanisme, il faut, avant d'en déduire, par extrapolation, que les écosystèmes sont préservés parce que la cosmologie les présente comme inclus dans le cercle de société, prendre en compte la réalité des pratiques, et non la « représentation » du milieu naturel. Si j'affirme que telle faune locale ne court aucun danger en raison d'une éthique cynégétique rigoureuse - on ne tue que pour manger -, j'affirme quelque chose qui n'a pas d'ancrage dans le réel, mais uniquement dans les représentations. Aussi, lorsqu'un équilibre faunistique est rompu, comme dans l'Uaçá, entraînant la prolifération du Caïman noir (Melanosuchus niger), la disparition progressive du Jacaretinga (Caiman crocodilus) ou de la Tracajá (Podocnemis unifilis), l'anthropologue ne peut se contenter d'invoquer « la Mère des caïmans » et se maintenir dans le décryptage cosmologique, hors de toute incursion dans le domaine de la réalité. Ces déséquilibres ont des implications écologiques, sociales, économiques, mais aussi légales s'agissant d'espèces protégées ou interdites à la vente. Les ONG environnementalistes désireuses de pénétrer l'univers des associations indigènes admettent la non-réciprocité des engagements et l'absence de formes de contractualisation (Ferreira, 2004; Araújo, 2009) ${ }^{4}$, ce qui rend vaine toute intervention dans ce domaine, la connaissance précise de la démographie des différentes espèces (humaine comprise) venant buter sur l'omission obligée et sur l'impossibilité d'agir (Kohler, 2008). Lorsque la contractualisation est invoquée, c'est sous la forme d'un « engagement moral » dont on sait pertinemment qu'il n'est susceptible d'aucune rétorsion ${ }^{5}$.

La santé des écosystèmes, telle qu'elle transparaît dans la carte qui nous a servi d'exemple (Fig. ci-contre), est donc davantage le fait d'une faible pression démographique que $d$ 'une "cosmologie fondamentalement respectueuse des écosystèmes ». Il faut donc, pour

\footnotetext{
${ }^{4}$ Cette problématique s'étend dorénavant aux «populations traditionnelles », puisqu'elles forment depuis 2007 une entité juridique à part entière dans la législation brésilienne (décret $\mathrm{n}^{\circ} 6040$ du 7 mars).

5 Les populations concernées étant généralement illettrées et insolvables, il est parfaitement vain d'invoquer le respect de cahiers des charges ou de bonnes pratiques de gestion comme condition à leur permanence dans les lieux, dans la mesure où aucune amende ne saurait être infligée et que l'on imagine mal mettre en œuvre, aujourd'hui, le déplacement forcé de populations rurales au motif qu'elles auraient contribué à l'extinction d'une espèce rare de perroquet.
} 
appréhender les dispositions d'une population à l'égard de son environnement, une heuristique des représentations qui établisse clairement la distinction entre ce qui relève de l'idéologie (les discours contextuels relatifs au développement durable, au respect de la nature, etc., c'est-à-dire des "structures structurées ») et ce qui relève de la cosmologie, ancrée dans l'histoire et la mémoire, tant individuelles que collectives (des «structures structurantes »).

\section{L'approche pluridisciplinaire comme moteur de l'enquête}

L'autre obstacle à une heuristique de la réalité dépouillée de préconçus idéologiques est, nous l'avons dit, la solitude de l'anthropologue sur le terrain, qui le prédispose à prendre fait et cause pour les populations enquêtées sans toutefois disposer d'une vue d'ensemble de la réalité. C'est le dilemme inhérent à " l'anthropologie impliquée »-anthropological advocacy -, dont Albert (1995, p. 15) pointe le danger : «Dès lors que [le projet] tend vers la conquête de droits fondamentaux de citoyenneté constamment déniés par les États, l'anthropologue ne peut, bien entendu, que souscrire à son propos et aux actions qui en découlent. Mais il ne saurait, en revanche, accepter que sa recherche se borne à ce cadre, aussi incontestable qu'il soit. »

Parce que l'abord anthropologique s'avère insuffisant à rendre compte, à lui seul, des impacts sur un milieu et des pressions exercées par une population humaine, à inventorier la flore et la faune ou à mesurer la toxicité de l'eau et des sols, la complexité du terrain exige la constitution d'équipes interdisciplinaires incluant, entre autres, des écologues et des géographes. Nous prendrons pour exemple de ce mode de travail et de constitution d'équipes le programme DURAMAZ, achevé en 2010, programme pluridisciplinaire et multisitué qui se proposait d'aborder la question des déterminants géographiques, économiques, démographiques et anthropologiques du développement durable en Amazonie brésilienne. Le choix des terrains, volontairement hétérogènes, incluait tant des territoires amérindiens que des fronts pionniers, des populations traditionnelles et des exploitations agro-industrielles, leur point commun étant d'avoir fait l'objet d'expériences de développement durable remontant au moins à une dizaine d'années. L'objectif était de mettre au point un système d'indicateurs suffisamment exhaustif (Droulers et Le Tourneau, 2010) et flexible pour permettre la prise en compte de données de natures très variées (éducation, santé, équipement, revenus, mais aussi trajectoires, activités, cadres géographiques et politiques, types paysagers [Guillaumet et al., 2009], biodiversité), ainsi qu'un système de transposition permettant des comparaisons synoptiques, propres à appréhender l'ensemble des facteurs influant sur la réussite des projets, tant sur le plan social que sur les plans économique et environnemental.

Dans le cadre du travail d'équipe, l'anthropologue doit se contraindre à traiter son matériau de manière à ce que les résultats de son enquête soient exploitables et pertinents pour d'autres disciplines, en analysant en priorité les significations locales des concepts qui accompagnent et justifient les politiques publiques. «Développement durable», « gouvernance», «globalisation », « société civile», sont autant de termes dont l'imprécision est une condition de leur applicabilité dans les politiques publiques; cela n'en fait pas pour autant des concepts anthropologiques, mais bien plutôt des « choses conceptuelles » à étudier localement, comme peuvent l'être les « choses culturelles » telles que le mariage ou la couvade (Sperber, 1996). Tandis que Diegues (1996) s'efforce de définir les populations traditionnelles en fonction d'éléments qui les naturalisent (Barretto Filho, 2006), il faut au contraire s'attacher à appréhender, au sein de ces communautés, un ensemble d'éléments, usages ou représentations, qui traduisent des formes d'attachement, d'enracinement, de cohésion, un ensemble de dispositions à l'égard du monde naturel qui laissent augurer une coexistence "durable», mais ne sauraient être tenues pour innées ou allant de soi.

Afin de respecter la logique pluridisciplinaire du programme, une série "d'indicateurs anthropologiques » fut forgée, après les enquêtes de terrain, en considérant différents aspects du capital social (Putnam, 1995) : formes de sociabilité, conception de la parenté, appartenances religieuses, mémoire collective, étape migratoire, cela afin de proposer une vue synoptique des indices de cohésion communautaire et d'ancrage territorial. Une autre série d'indicateurs venait compléter la première, cette fois relativement à l'attachement ou à la familiarité au milieu (Reichel-Dolmatoff, 1976; Dugast, 2002) : toponymie, connaissance de l'histoire locale, connaissances botaniques, créatures surnaturelles, etc. Ces indicateurs valent évidemment si l'on considère les communautés au sens wébérien ${ }^{6}$ du terme - nous les qualifierons schématiquement de «traditionnelles » (Encadré).

L'enquête anthropologique, par sa capacité à distinguer, dans l'analyse des propos, ce qui relève d'un discours spontané ou contraint, permet d'appréhender la disposition des habitants à entrer dans un cycle vertueux d'équilibre et de pérennité. Il en va du «développement durable » comme de la démocratie et du libre échange : une approche strictement économique associe

\footnotetext{
${ }^{6}$ Selon Weber, le terme de communauté qualifie une collectivité envers laquelle on se sent des devoirs structurés par un sentiment d'appartenance, qui sous-tendent des liens de solidarité. Mais on peut aussi l'appréhender de manière dynamique, comme réseaux multiscalaires et multidimensionnels (Berkes, 2004).
} 


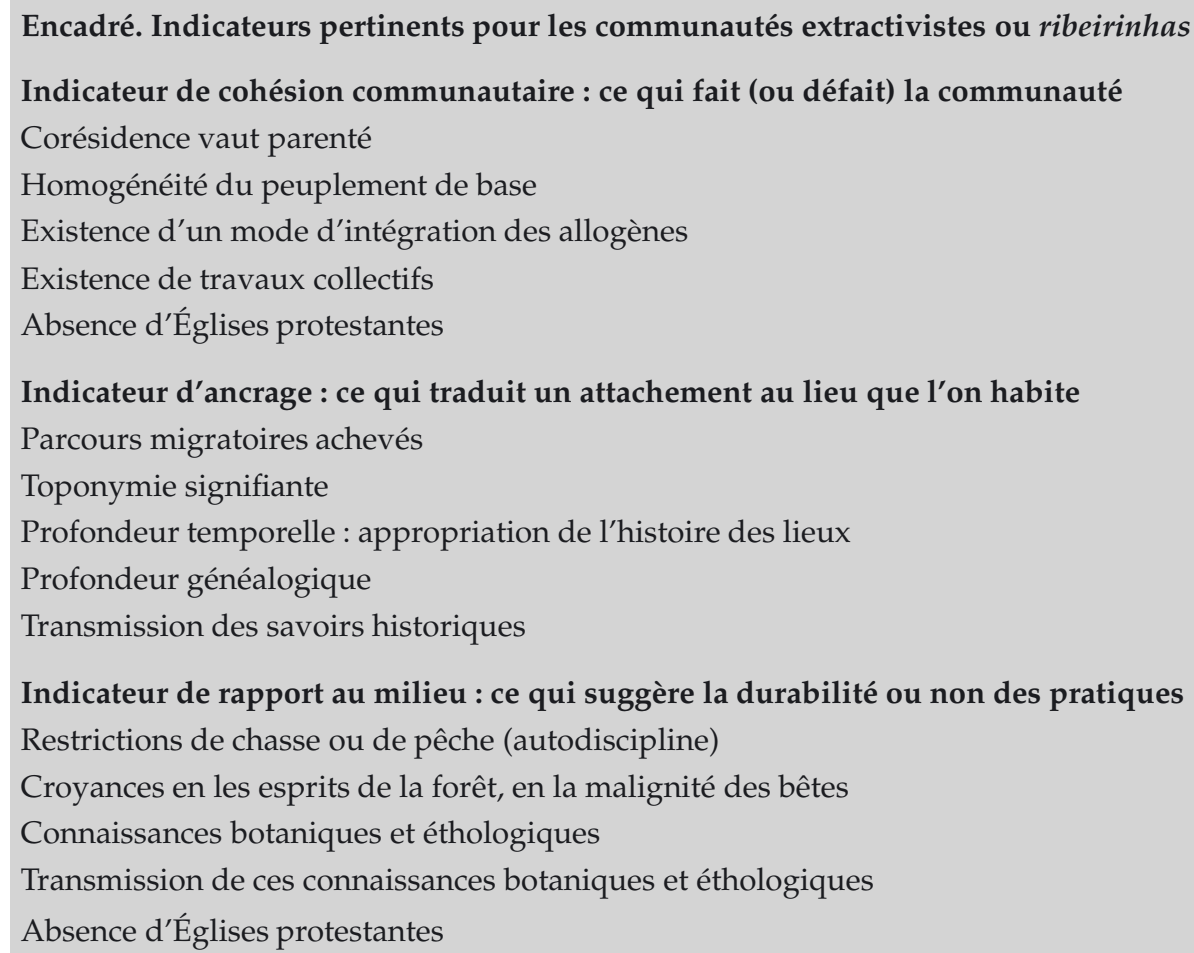

la libéralisation des échanges à une progressive entrée des nations dans l'arène des pays démocratiques. Mais on peut se demander «si, à l'inverse, ce n'est pas plutôt la qualité des institutions et de la vie démocratique, la force du capital social qui conditionnent la croissance économique » (Caillé, 2006, p. 10). La définition retenue, dans le cadre de DURAMAZ, est celle du sociologue James Coleman (1988, p. S116), qui pose le capital social comme étant la «valeur collective de toutes les normes et relations sociales permettant la coordination d'actions en vue d'atteindre des objectifs communs ». Ce concept possède une valeur centrale dans les études récentes portant sur la renégociation des communs (Brondizio et al., 2009).

Appliquons ce constat aux sociétés traditionnelles (englobant, donc, les populations indigènes et traditionnelles proprement dites) : ce qui permet à une telle société d'accéder à un meilleur patron d'existence ne se limite pas à la somme de projets que l'on y investit. C'est aussi le capital social dont elle dispose, la qualité des relations humaines, l'attachement à un cadre de vie, la solidarité qui s'exprime dans les travaux et les jours, un ensemble d'éléments, donc, qui sont autant de signes que cette microsociété n'a pas pour unique objectif de révolutionner son mode d'existence pour accéder aux infrastructures. C'est l'étude comparée de deux communautés au profil similaire (Kohler et al., 2009), toutes deux situées en Amapá, qui a permis de dégager cet axiome : toute chose étant égale par ailleurs, la réussite des projets de développement durable est conditionnée par la cohésion communautaire face aux forces centrifuges provoquées par l'afflux d'argent et de projets. Ce constat s'applique aux sociétés indigènes confrontées à l'ascension d'une élite politique détentrice de réseaux d'alliance avec les ONG indigénistes et les édiles régionaux ${ }^{7}$.

\section{Conclusions}

La thèse que nous défendons dans cet article, fondée sur la position de Lévi-Strauss exposée en exergue, est la suivante : une politique orientée vers la préservation de la biodiversité ne peut se limiter à déléguer aux sociétés traditionnelles, ou se réclamant de la tradition, le soin de gérer des espaces protégés. Ce n'est ni leur rôle ni leur vocation, comme les tentatives de réintroduction de l'ours dans le haut Béarn, entravées par le caractère traditionnel du pastoralisme, l'ont tristement illustré (Mermet, 2002 ; Mermet et Benhammou, 2005). Il faut donc établir un distinguo entre ce qui relève de la préservation de la biodiversité et ce qui n'en relève pas.

Par ailleurs, la création d'aires protégées, habitées ou non, agit bien souvent comme un facteur d'attraction pour les populations (Wittemyer et al., 2008), générant une forte pression démographique : ces zones sont rendues attrayantes par les emplois directs ou indirects liés à l'aire protégée, mais fonctionnent aussi comme des

\footnotetext{
${ }^{7}$ Un épisode de crise convulsive dans le village Galibi-Marworno de Kumarumã pouvait être interprété-c'était notre hypothèse - comme l'expression d'un malaise suscité par de graves disparités sociales en voie de consolidation (Kohler, 2010).
} 
viviers ou des pépinières, grâce aux ressources qui essaiment en leurs abords (gibier, bois de feu, herbes médicinales... ). Dans le cas brésilien, la création d'une aire protégée s'accompagne de garanties foncières octroyées à des populations locales qui, sans ce recours, eussent été vraisemblablement spoliées par l'avancée de fronts de colonisation (Ferreira, 2004; Pinton et Grenand, 2007). Les conditions améliorées de santé et d'éducation et les infrastructures nouvelles entraînent quasi mécaniquement une croissance démographique accélérée, tant par la natalité que par les retours au pays. Les pressions sont d'abord cantonnées à la zone "tampon », jusqu'au seuil de nonrenouvellement. La pénétration progressive de l'aire protégée survient alors comme une fatalité, et accroît sa vulnérabilité jusqu'au seuil critique de dévastation.

En conséquence, la création d'aires protégées habitées, ou l'octroi de droits spécifiques aux populations occupant traditionnellement des zones riches en ressources renouvelables, peut apparaître comme une solution préférable, à moyen terme, à la sanctuarisation de vastes étendues autour desquelles s'accentue la pression démographique (Schwartzmann et al., 2000; Nepstad et al., 2006). Les populations incluses dans le périmètre sont alors mieux à même de faire respecter le droit - c'est le principe des «Unités de conservation d'usage direct » au Brésil ${ }^{8}$. Les lois s'appliquent mieux si des humains, et non des jaguars, se déclarent lésés.

Mais l'attitude des populations concernées à l'égard de leur environnement, les conditions socioéconomiques qui leur permettront d'améliorer leur train d'existence sans intensifier la ponction exercée, sont des facteurs d'évaluation fondamentaux pour tout projet lié au « développement durable». Il faut pour cela une approche sereine et objective, et non des pétitions de principe posant que la diversité culturelle est garante de la diversité biologique.

Aussi, avant de tenir pour acquis que les populations indigènes ou traditionnelles sont « constitutivement » respectueuses des écosystèmes, faut-il se doter d'une heuristique des représentations qui commencerait par distinguer ce qui procède d'une vision cosmologique (historique, mythologique et partagée par le groupe) et ce qui dérive d'un système idéologique produit par le contexte particulier des politiques de protection environnementale alliées aux projets de développement durable. Une fois équipé d'une telle méthode, il convient de ne pas négliger les données issues de l'observation, concernant l'usage effectif qui est fait des ressources naturelles. Le travail en équipe nous paraît seul à même de fournir un ensemble exhaustif d'éléments d'appréciation, tout en préservant l'anthropologue d'une implication forcée dans les dynamiques de revendication.

${ }^{8}$ Loin $^{\circ} 9985$ du 18 juillet 2000 ; décret n 4340 du 22 août 2002.

\section{Remerciements}

Cet article n'aurait pas vu le jour sans le soutien du CREDA, UMR7227 (CNRS-Université Paris 3), qui m'a permis d'aborder sous de multiples angles la problématique des aires protégées en Amazonie. Je remercie également le Núcleo de História do Indígena e do Indigenismo (NHII) de 1'Université de São Paulo, en particulier Dominique Gallois et Lux Vidal, qui m'ont ouvert l'accès aux terrains de l’Uaçá.

\section{Références}

Albert, B., 1995. Anthropologie appliquée ou anthropologie «impliquée »? Ethnographie, minorités, et développement, in Baré, J.-F. (Ed.), Les Applications de l'anthropologie : un essai de réflexion collective depuis la France, Karthala, 87-118.

Álvares, M.M., 1992. Yãmiy, os espíritos do canto : a construção da pessoa na sociedade Maxakali. Dissertação de Mestrado, Unicamp, Belo Horizonte.

Appadurai, A., 2003. Diversité et développement durable, in Unesco, Diversité culturelle et biodiversité pour un développement durable : table ronde de haut niveau organisée conjointement par l'Unesco et le PNUE le 3 septembre 2002 à Johannesburg à l'occasion du Sommet mondial pour le développement durable, 16-19.

Araújo, R., 2009. De la « communauté » aux «populations traditionnelles », Nuevo Mundo Mundos Nuevos, Coloquios : http://nuevomundo.revues.org/index56593.html.

Balée, W., 1994. Footprints of the Forest, New York, Columbia University Press.

Barbault, R., 2005. Biodiversité, écologie et société, Écologie E Politique, 30, 27-40.

Barretto Filho, H.T., 2006. Populações tradicionais : introdução à crítica da ecologia política de uma noção, in Adams, C., Murrieta, R., Neves, W. (Eds), Sociedades caboclas amazônicas : modenidade e invisibilidade, São Paulo, Annablume, 109-144.

Belleau, J.-P., 2007. Sociologie du mouvement indien au Brésil : une approche à partir de la théorie $d u$ processus politique. Thèse de doctorat en sociologie, Université Paris 3, IHEAL, Paris.

Berkes, F., 2004. Rethinking community-based conservation, Conservation Biology, 18, 3, 621-630.

Brondízio, E.S., Ostrom, E., Young, O.J., 2009. Connectivity and the governance of multilevel social-ecological systems: The role of social capital, Annual Review of Environment and Resources, 34, 253-278.

Broswimmer, F.J., 2003. Écocide : une brève histoire de l'extinction en masse des espèces, Paris, Parangon.

Caillé, A., 2006. Préface, in Bevort, A., Lallement, M. (Eds), Le Capital social : performance, équité et réciprocité, Paris, La Découverte.

Caillon, S., Degeorges, P., 2005. Biodiversité(s), quand les frontières entre culture et nature s'effacent, Écologie $\mathcal{E}$ Politique, 30, 85-95.

Chapin, M., 2004. A challenge to conservationists, World Watch, $17,6,12-31$.

Chatterjee, S., 1997. The Rise of Birds: 225 Million Years of Evolution, Baltimore, The John Hopkins University Press.

Clastres, H., 1975. La Terre sans mal : le prophétisme tupi-guarani, Paris, Le Seuil. 
Coleman, J.S., 1988. Social capital in the creation of human capital, American Journal of Sociology, 94, Supp., S95-S120.

Conklin, B., Graham, L., 1995. The shifting middle ground: Amazonian Indians and eco-politics, American Anthropologist, 97, 695-710.

Cunha, M.C. da, Almeida, M.W.B. de, 2000. Indigenous people, traditional people, and conservation in the Amazon, Daedalus: Journal of the American Academy of Arts and Sciences, 129, 2, 315-338.

Descola, P., 2005. Par-delà nature et culture, Paris, Gallimard.

Diamond, J., 2006. Effondrement : comment les sociétés décident de leur disparition ou de leur survie, Paris, Gallimard.

Diegues, A.C., 1996. O Mito Moderno da Natureza Intocada, São Paulo, Editora Hucitec Ltda.

Dove, M.R., 2006. Indigenous people and environmental politics, Annual Review of Anthropology, 35, 191-208.

Droulers, M., Le Tourneau, F.M., 2010. L'Amazonie et le développement durable : leçons du programme DURAMAZ, Paris, Belin.

Dugast, S., 2002. Mode d'appréhension de la nature et gestion patrimoniale du milieu, in Cormier-Salem, M.-C., Juhé-Beaulaton, J., Boutrais, J., Roussel, B. (Eds), Patrimonialiser la nature tropicale : dynamiques locales, enjeux internationaux, Paris, IRD Éditions, 31-77.

Dumoulin-Kervran, D., 2006. Les «terres indiennes pour la conservation ", un dispositif-clé du néo-indigénisme international, in Gros, C., Strigler, M.-C. (Eds), Être Indien dans les Amériques, Paris, Éditions de l'Institut des Amériques, 91-104.

Ehrlich, P.R., Pringle, R.M., 2008. Where does biodiversity go from here? A grim business-as-usual forecast and a hopeful portfolio of partial solutions, PNAS, 105, Supp. 1, 11579-11586.

Ferreira, L. da Costa, 1999. Conflitos sociais contemporâneos : considerações sobre o ambientalismo brasileiro, Ambiente $\mathcal{E}$ Sociedade, 5, 35-54.

Ferreira, L. da Costa, 2004. Dimensões humanas da biodiversidade : mudanças sociais e conflitos em torno de áreas protegidas no Vale do Ribeira, SP, Brasil, Ambiente $\mathcal{E}$ Sociedade, 7, 1, 47-66.

Gifford, H.M., Fogel, M.L., Magee, J.W., Gagan, M.K., Clarke, S.J., Johnson, B.J., 2005. Ecosystem collapse in Pleistocene Australia and a human role in megafaunal extinction, Science, 309, 5732, 287-290.

Gras, A., 2004. La socio-anthropologie, une critique radicale de l'évolutionnisme, Socio-anthropologie, 14 ; mis en ligne le 15 mai 2005, URL : http://socio-anthropologie.revues.org/ index373.html (consulté le 25 juin 2010).

Grenier, C., 2008. La gestion de la biodiversité dans des régions à forte géodiversité : les cas du Corcovado (Costa Rica), des Galápagos (Équateur) et de Rapa Nui (Chili), in Héritier, S., Laslaz, L. (Eds), Les Parcs nationaux dans le monde : protection, gestion et développement durable, Paris, Ellipses, 123-145.

Guillaumet, J.-L., Laques, A.E., Léna, P., Robert, P. de, 2009. La Spatialisation de la biodiversité : pour la gestion durable des territoires, Paris, IRD Éditions.

Hames, R., 2007. The ecologically noble savage debate, Annual Review of Anthropology, 36, 177-190.

Harmon, D., 1996. Losing species, losing languages: Connections between biological and linguistic diversity, Southwest Journal of Linguistics, 15, 89-108.

Harmon, D., Maffi, L., 2002. Are linguistic and biological diversity linked?, Conservation Biology in Practices, 3, 1, 26-27.
Hayashida, F., 2005. Archaeology, ecological history, and conservation, Annual Review of Anthropology, 34, 43-65.

Hunt, T., Lipo, C.P., 2010. Ecological catastrophe, collapse, and the myth of "Ecocide" on Rapa Nui (Easter Island), in McAnany, P.A., Yoffee, N., Questioning Collapse: Human Resilience, Ecological Vulnerability, and the Aftermath of Empire, New York, Cambridge University Press.

Ingold, T. (Ed.), 1994. What is an Animal? , London and New York, Routledge.

Kelly, R., Prasciunas, M., 2007. Did the ancestors of Native Americans cause animal extinctions in late-Pleistocene North America, in Harkin, M., Lewis, D. (Eds), Native Americans and the Environment: Perspectives on the Ecological Indian, Lincoln, University of Nebraska Press, 95-122.

Kohler, F. (Ed.), 2007. Stéréotypes culturels et constructions identitaires, Tours, Presses universitaires François-Rabelais.

Kohler, F., 2008. Effets collatéraux des programmes de conservation sur le littoral brésilien, Études rurales, 181, 75-88.

Kohler, F., 2010. Chamanisme et politique dans l’Uaçá (bassin de l'Oyapock, Amapá), Anthropologie et société, 34, 1, 13-32.

Kohler, F., Greissing, A., Le Tourneau, F.-M., 2009. Deux maisons, égales en dignité : une approche anthropologique des déterminants du développement durable en contexte amazonien "traditionnel », Développement durable et territoires : http://developpementdurable.revues.org/index8173.html.

Krech, S., 1999. The Ecological Indian: Myth and History, New York and London, W.W. Norton.

Latour, B., 1991. Nous n'avons jamais été modernes : essai d'anthropologie symétrique, Paris, La Découverte.

Latour, B., 1999. Politiques de la nature, Paris, La Découverte / Sciences humaines et sociales.

Lévi-Strauss, C., 1971. Race et culture, discours inaugural de l'Année internationale de la lutte contre le racisme, prononcé à l'Unesco, Paris.

Lévi-Strauss, C., 1983. Le Regard éloigné, Paris, Plon.

Mermet, L., 2002. Homme ou vie sauvage? Société locale ou bureaucratie centrale? Faux dilemmes et vrais rapports de force, Les Annales des Mines / Responsabilité E Environnement, 28, 13-20.

Mermet, L., Benhammou, F., 2005. Prolonger l'inaction environnementale dans un monde familier : la fabrication stratégique de l'incertitude sur les ours du Béarn, Écologie $\mathcal{E}$ politique, 31, 121-136.

Morin, F., 1992. Revendications et stratégies politiques des organisations indigènes amazoniennes, Cahiers des Amériques latines, 13, 75-85.

Nadasdy, P., 2005. Transcending the debate over the ecologically noble Indian: Indigenous peoples and environmentalism, Ethnohistory, 52, 291-331.

Nepstad, D., Schwartzmann, S., Bamberger, B., Santilli, M., Ray, D., Schlesinger, P., Lefebvre, P., Alencar, A., Prinz, E., Fiske, G., Rolla, A., 2006. Inhibition of Amazon deforestation and fire by parks and indigenous lands, Conservation Biology, 20, 1, 65-73. 
Pinton, F., Grenand, P., 2007. Savoirs traditionnels, populations locales et ressources globalisées, in Aubertin, C., Pinton, F., Boisvert, V. (Eds), Les Marchés de la biodiversité, Paris, IRD Éditions, 165-194.

Posey, D.A., 1985. Indigenous management of tropical forest ecosystems: The case of the Kayap'o Indians of the Brazilian Amazon, Agroforestry Systems, 3, 2, 139-158.

Posey, D.A., Dutfield, G., 1996. Beyond Intellectual Property: Toward Traditional Resource Rights for Indigenous Peoples and Local Communities, Ottawa, International Development Research Center.

Putnam, R., 1995. Bowling alone: America's declining social capital, Journal of Democracy, 6, 1.

Rappaport, R., 1968. Pigs for the Ancestors, New Haven (CT), Yale University Press.

Reichel-Dolmatoff, G., 1976. Cosmology as ecological analysis, Man, 11, 3, 307-318.

Schaeffer, J.-M., 2007. La Fin de l'exception humaine, Paris, Gallimard.

Scheps, R. (Ed.), 1993. La Science sauvage : des savoirs populaires aux ethnosciences, Paris, Le Seuil.

Schwartzman, S., Moreira, A., Nepstad, D., 2000. Rethinking tropical forest conservation: Perils in parks, Conservation Biology, 14, 5, 1351-1357.

Sperber, D., 1996. La Contagion des idées, Paris, Odile Jacob.

Steadman, D.W., Martin, P.S., MacPhee, R.D.E., Jull, A.J.T., McDonald, H.G., Woods, C.A., Iturralde-Vinent, M., Hodgins, G.W.L., 2005. Asynchronous extinction of late Quaternary sloths on continents and islands, PNAS, 102, 33, 11763-11768.

Stoczkowski, W., 1994. Anthropologie naïve, anthropologie savante: de l'origine de l'homme, de l'imagination et des idées reçues, Paris, CNRS Éditions.
Surovell, T., Waguespack, N., Brantingham, P., 2005. Global archaeological evidence for proboscidean overkill, PNAS, 102, $17,6231-6236$.

True, C., Field, J., Dortch, D., Charlels, B., Wroe, S., 2005. Prolonged existence of humans and megafauna in Pleistocene Australia, PNAS, 102, 23, 8381-8385.

Tudge, C., 1996. The Time before History: 5 Million Years of Human Impact, New York, Scribner.

Unesco, 2003. Diversité culturelle et biodiversité pour un développement durable : table ronde de haut niveau organisée conjointement par l'Unesco et le PNUE le 3 septembre 2002 à Johannesburg à l'occasion du Sommet mondial pour le développement durable, Paris, Unesco. Document téléchargeable à l'URL : http://www.unesco.org/new/fr/unesco/resources/ publications/unesdoc-database/

Vigne, J.-D., 1999.The large "true" Mediterranean islands as a model for the Holocene human impact on the European vertebrate fauna? Recent data and new reflections, in Benecke, N. (Ed.), The Holocene History of the European Vertebrate Fauna: Modern Aspects of Research. Workshop, 6th-9th April 1998, Berlin, Rahden, VML, 295-322.

Viveiros de Castro, E., 2002. A Inconstância da alma selvagem e outros ensaios de antropologia, São Paulo, Cosac Naify.

Viveiros de Castro, E., 2008. O Brasil é grande, mas o mundo é pequeno, Jornal O Rebate, 2 juillet.

West, P., Igoe, J., Brockington, D., 2006. Parks and peoples: The social impact of protected areas, Annual Review of Anthropology, 35, 251-277.

Wittemyer, G., Elsen, P., Bean, W.T., Coleman, A., Burton, O., Brashares, J.S., 2008. Accelerated human population growth at protected area edges, Science, 321, 5885, 123-126.

Reçu le 22 janvier 2010. Accepté le 1 ${ }^{\text {er juillet } 2010 .}$ 